\title{
Residual Thromboembolic Material in Cerebral Arteries after Endovascular Stroke Therapy Can Be Identified by Dual-Energy CT
}

\author{
A.E. Grams, M. Knoflach, R. Rehwald, J. Willeit, M. Sojer, E.R. Gizewski, and B. Glodny
}

\begin{abstract}
BACKGROUND AND PURPOSE: Dual-energy CT features the opportunity to differentiate among up to 3 different materials because the absorption of $x$-rays depends on the applied tube voltage and the atomic number of the material. For example, it is possible to distinguish between blood-brain barrier disruption and an intracerebral hemorrhage following treatment for a stroke. The aim of this study was to evaluate whether dual-energy CT is capable of distinguishing intra-arterial contrast agent from residually clotted vessels immediately after endovascular stroke therapy.
\end{abstract}

MATERIALS AND METHODS: Sixteen patients ( 9 women, 7 men; mean age, $63.6 \pm 13.09$ years) were examined. Measurements were made on the postinterventional dual-energy CT virtual noncontrast, iodine map, and "weighted" brain window (weighted dual-energy) series. Postinterventional conventional angiography was used as the criterion standard method.

RESULTS: A residual clot was found in 10 patients. On the virtual noncontrast series, the Hounsfield attenuation of the clotted arteries was higher than that in the corresponding perfused contralateral arteries $(53.72 \pm 9.42 \mathrm{HU}$ versus $41.64 \pm 7.87 \mathrm{HU} ; P<.05)$. The latter had higher absorption values on the weighted dual-energy series than on the virtual noncontrast series $(49.37 \pm 7.44 \mathrm{HU}$ versus $41.64 \pm 7.87 \mathrm{HU} ; P<$ .05). The sensitivity for the detection of a residual clot was $90 \%$; the specificity was $83.3 \%$, and the accuracy was $87.5 \%$. Interrater agreement was $\operatorname{good}(\kappa=0.733)$.

CONCLUSIONS: Dual-energy CT may be valuable in the detection of clot persistence or early re-thrombosis without the necessity of additional contrast administration. However, its relevance for the prediction of outcomes remains to be determined in further studies.

ABBREVIATIONS: $\mathrm{Cl}=$ confidence interval; $\mathrm{DECT}=$ dual-energy $\mathrm{CT} ; \mathrm{DEw}=$ weighted dual-energy series; $\mathrm{IM}=$ iodine map series; $\mathrm{VNC}=$ virtual noncontrast series

I specialized centers, endovascular stroke therapy is a method frequently used in addition to or instead of intravenous thrombolysis. In recent decades, various intra-arterial treatment methods such as intra-arterial thrombolysis, clot aspiration, and stent retrievers have been introduced. ${ }^{1,2}$ During the intervention, a contrast agent must be administered; the diagnostic value of a postinterventional CT is therefore limited because hyperattenuated hemorrhage cannot always be differentiated from hyperattenuated intraparenchymal contrast staining due to blood-brain barrier disruption. It has been shown that this dilemma can be overcome with dual-energy CT (DECT). DECT is a quite recent innovation in CT imaging, in which datasets are acquired with 2

Received November 29, 2014; accepted after revision January 26, 2015.

From the Departments of Neuroradiology (A.E.G., E.R.G.), Neurology (M.K., J.W., M.S.), and Radiology (R.R., B.G.), Medical University of Innsbruck, Innsbruck, Austria Please address correspondence to Astrid Ellen Grams, MD, Department of Neuroradiology, Medical University of Innsbruck, Anichstr 35, 6020 Innsbruck, Austria; e-mail: astrid.grams@googlemail.com, astrid.grams@i-med.ac.at

http://dx.doi.org/10.3174/ajnr.A4350 different tube voltages, typically with $80 \mathrm{kV}$ or 100 and $140 \mathrm{kV} .^{3-5}$ Individual tube settings are adapted so that the overall dose does not exceed the dose of a comparable conventional CT acquired with 1 tube voltage. Some studies even suggest that a dose reduction can be achieved with DECT compared with conventional CT. ${ }^{6,7}$ In principle, the absorption of $\mathrm{x}$-rays depends on the tube voltage and the atomic number of a tissue. ${ }^{8-11}$ While for low or medium voltages, the photoelectric effect is more likely to be responsible for the absorption of radiation, for medium voltages Compton scattering is more often responsible. ${ }^{12,13}$ The photoelectric effect and Compton scattering are related in different ways to the energy of the radiation and the mass of the material radiated. While the probability of the occurrence of a photoelectric effect increases with the fourth power of the atomic number and decreases with the third power of the energy, the probability of the occurrence of Compton scattering increases only linearly with the atomic number of the radiated element and decreases linearly with radiation energy. ${ }^{12}$ These phenomena allow up to 3 different materials to be distinguished. ${ }^{12}$ The main application is 
Table 1: Relevant information about the included patients

\begin{tabular}{|c|c|c|c|c|c|c|c|}
\hline No. & Sex & Age (yr) & Clot & $\begin{array}{l}\text { Intravenous } \\
\text { Thrombolysis }\end{array}$ & Residual Clot & $\begin{array}{l}\text { Intra-Arterial } \\
\text { Thrombolysis }\end{array}$ & $\begin{array}{l}\text { Hematocrit } \\
\text { (I/I) }\end{array}$ \\
\hline 1 & Female & 79 & BA & Yes & Right P1 & Yes & 0.276 \\
\hline 2 & Male & 79 & BA & No & Left P2 & Yes & 0.346 \\
\hline 3 & Male & 50 & Right MCA & Yes & M2 & Yes & 0.441 \\
\hline 4 & Female & 73 & Left MCA & No & M2 & Yes & 0.401 \\
\hline 5 & Female & 58 & Right MCA & No & M3 & No & 0.429 \\
\hline 6 & Male & 63 & Left MCA & Yes & M3 & Yes & 0.438 \\
\hline 7 & Female & 77 & Right MCA & Yes & M2 & Yes & 0.419 \\
\hline 8 & Female & 51 & Right MCA & Yes & M3 & Yes & 0.413 \\
\hline 9 & Female & 62 & Left MCA & No & M3 & Yes & 0.354 \\
\hline 10 & Female & 48 & Right MCA & Yes & M3 & No & 0.4 \\
\hline 11 & Female & 73 & Right MCA & Yes & No & Yes & 0.315 \\
\hline 12 & Male & 58 & Right MCA & Yes & No & Yes & 0.41 \\
\hline 13 & Female & 48 & $\mathrm{BA}$ & Yes & No & Yes & 0.36 \\
\hline 14 & Male & 50 & $\mathrm{BA}$ & Yes & No & No & 0.401 \\
\hline 15 & Male & 60 & Right MCA & No & No & Yes & 0.384 \\
\hline 16 & Male & 89 & Right MCA & Yes & No & Yes & 0.431 \\
\hline
\end{tabular}

Note:-BA indicates basilar artery.

for the differentiation between hemorrhagic transformation and contrast staining in the brain after recent intravascular contrast application. ${ }^{13-19}$ After neuroendovascular therapy, important questions regarding possible hemorrhage, swelling, or infarction can be answered reliably with this method. ${ }^{18}$ However, this method is also widely applied to differentiate various tissues in other regions of the body. ${ }^{20-23}$

The hyperattenuated artery sign is an accepted tool for detecting a clotted vessel in the diagnostic management of acute stroke on unenhanced CT scans. ${ }^{24,25}$ However, other factors that influence vascular attenuation, mainly an increased hematocrit level, must be taken into account when interpreting vascular attenuation. ${ }^{26,27}$ Postinterventionally, residual contrast agent remaining in the vessel is the greatest factor affecting vascular attenuation. It can prevent the correct diagnosis of residual or new clots on a CT scan because the increased vascular attenuation due to contrast agent cannot be distinguished from genuine clots that usually appear hyperattenuated. ${ }^{14}$ However, DECT could, theoretically at least, make this distinction possible because the iodine still remaining in the vessel could be quantified and, by using voxels, subtracted from the absorption values found. ${ }^{14,18}$ However, this technique has never been investigated with the focus on small residual clots following endovascular stroke therapy. Therefore, the aim of the present study was to discover whether residual intra-arterial clots after endovascular stroke therapy can be detected with DECT by using conventional angiography as the criterion standard.

\section{MATERIALS AND METHODS}

This study was approved by the local ethics review board. From October 2013 to October 2014, 16 patients (9 women and 7 men) with a mean age of $63.6 \pm 13.09$ years (range, $48-89$ years) who received endovascular therapy after an ischemic stroke due to intracranial vascular occlusion from an intra-arterial clot were examined. A preinterventional conventional CT scan $(n=15)$ had been performed by using different scanners because the patients were transferred from other hospitals or from different locations of the hospital campus. Twelve patients had an occlusion of the middle cerebral artery; and 4 had an occlusion of the basilar artery. Most patients received intravenous thrombolysis with recombinant tissue plasminogen activator before endovascular therapy. The reasons for the others not receiving thrombolysis were due to therapy with anticoagulants $(n=3)$, recent stroke $(n=1)$, or a recent surgical procedure $(n=1)$. Endovascular therapy was performed with the patient under either general or local anesthesia. In all patients, recanalization was performed by using a stent retriever. Some patients (Table 1) received additional intra-arterial thrombolysis. Demographic data of the 16 patients, sex, age, initial clot location, information about performed intravenous or intra-arterial thrombolysis, residual clot localization, and hematocrit values, are presented in Table 1.

After endovascular therapy, patients were immediately transferred to the DECT scanner (Somatom Definition Flash; Siemens, Erlangen, Germany). The postinterventional unenhanced DECT scan was performed within no more than 1 hour after finalization of the endovascular therapy, in most cases earlier, to rule out hemorrhage or infarct demarcation requiring further treatment such as decompressive craniectomy. The DECT datasets were acquired with both tubes in parallel. The tube parameters of the first tube were $100 \mathrm{kV}$ and $360 \mathrm{~mA}$; the second tube was operated at $140 \mathrm{kV}$ and $360 \mathrm{~mA}$. The other parameters were the same for both tubes: Pitch was 0.45 , the acquisition mode was incremental with $32 \times 0.6 \mathrm{~mm}$ collimation, section thickness was $4 \mathrm{~mm}$, and scan FOV was $200 \mathrm{~mm}$ in each. The following datasets were reconstructed secondarily: brain window weighted dual-energy series (DEw) in axial planes with a weighting of $50 \%$ for the $100 \mathrm{kV}$ and $50 \%$ for the $140 \mathrm{kV}$ series (section thickness, $4 \mathrm{~mm}$; increment, 4 mm; kernel, H30f medium smooth; window, base orbit; FOV, 200 $\mathrm{mm})$. From the data from each of the 2 tubes, series were calculated with the following parameters: 1-mm section thickness, 0.8-mm increment, H40f medium kernel, base orbit window, 200-mm FOV. Using the Brain Hemorrhage postprocessing software (Siemens), we constructed virtual noncontrast (VNC) and iodine map (IM) series on a dedicated workstation (syngo.CT Workplace 2012B; Siemens).

Attenuation measurements were performed by using ROIs, after maximum magnification in PACS software on a dedicated 


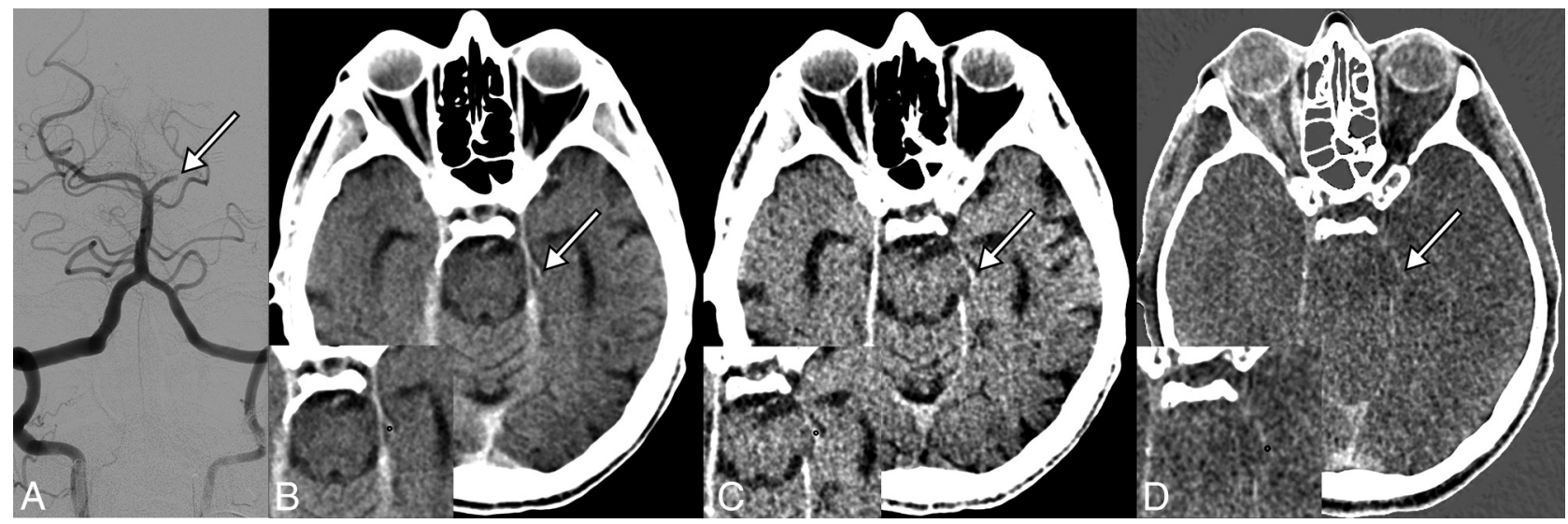

FIG 1. Residual clot (arrow) in the left P2 segment on conventional angiography $(A)$ and in the different dual-energy series: weighted dual-energy $(B)$, virtual noncontrast $(C)$, and iodine map $(D)$. The arrows indicate the hyperattenuated residual clots in the DEw and the VNC series. No clot is visible in the IM series. Note demonstration of ROI measurements in the residual clots after magnification in the left lower image sections.

Table 2: Attenuation measurements on the different series with mean values, SDs, upper/ lower $95 \%$ Cls of rater 1 , and results from Wilcoxon tests between residual clots and perfused vessels in the 10 patients with residual clots

\begin{tabular}{lcccc}
\hline & \multicolumn{4}{c}{ Hounsfield Attenuation (Mean) } \\
\cline { 2 - 5 } & CT & DEw & VNC & IM \\
\hline Clots & $58.88 \pm 10.98$ & $50.50 \pm 10.08$ & $53.72 \pm 9.42$ & $16.02 \pm 12.07$ \\
Lower/upper 95\% Cl of mean & $55.05 / 70.66$ & $43.29 / 57.71$ & $46.98 / 60.46$ & $7.39 / 24.65$ \\
Vessels & $43.35 \pm 5.54$ & $49.37 \pm 7.44$ & $41.64 \pm 7.87$ & $10.88 \pm 11.35$ \\
Lower/upper 95\% Cl of mean & $39.5 / 47.72$ & $42.37 / 53.71$ & $33.17 / 44.79$ & $10.55 / 15.13$ \\
$P$ values (Wilcoxon) & $.0091^{\mathrm{a}}$ & .4316 & $.0020^{\mathrm{a}}$ & .8457 \\
\hline
\end{tabular}

${ }^{a}$ Significant difference.

workstation (Impax EE R20 XIV v20140703_1249; Agfa-Gevaert, Mortsel, Belgium) by an experienced board-certified neuroradiologist (A.E.G.) and an experienced board-certified radiologist (B.G.).

In the preinterventional CT series, measurements were performed in the clotted vessels on the basis of the data from CT or conventional angiography and in the corresponding perfused segment of the MCA (M1 segment) on the contralateral side. If the basilar artery was occluded, the right middle cerebral artery was used for comparison. The same datasets were then blinded by removing all patient data including information on the clinical symptoms. Four months later, they were shown to the 2 raters in random order for identification of clot positions.

In the postinterventional DECT examination, measurements were performed within the residual clots known from a conventional angiogram only in the patients with residual clots (Fig $1 A$ ) and in the perfused contralateral MCA (M1 segment) in all patients on the different DEw (Fig 1B), VNC (Fig 1C), and IM (Fig $1 D)$ series. If residual clots were no longer present, measurements were made only in the contralateral MCA.

The postinterventional DECT datasets were also blinded as described above and were presented to the 2 raters 4 months later in random order for identification of the positions of residual clots.

Descriptive statistics such as mean values and SDs were compiled with the Excel software (Microsoft Office 2013; Microsoft, Redmond, Washington). Due to the small sample size, only nonparametric tests not requiring normal distribution were used. The Wilcoxon signed rank test was applied for matched-pairs group comparisons. Correlation analyses were performed by using the Spearman method. Sensitivity, specificity, and positive and negative predictive values regarding the detection of clots in the preinterventional CT and of residual clots in the postinterventional CT were calculated by using the data collected by the neuroradiologist (A.E.G.). To evaluate the interrater variability with respect to the correct localization of clots or residual clots, we determined a Cohen $\kappa$ for each. ${ }^{28}$ All these calculations were made with the statistics software GraphPad Prism, Version 6 (GraphPad Software, San Diego, California) and GraphPad on-line (www.graphpad.com). The interrater variability with respect to the agreement of the measured absorption values was evaluated by using the intraclass correlation coefficient as proposed by Shrout and Fleiss. ${ }^{29}$ A $P<.05$ was significant.

\section{RESULTS}

Descriptive data, mean attenuation, SDs, and $P$ values for the preinterventional CT and the different postinterventional DECT series and vascular segments are given in Table 2.

In the preinterventional conventional CT scan, the clotted arteries were significantly more attenuated than the perfused arteries. In 8 of 9 cases (88.9\%), clotted arteries could be identified by the blinded reader due to pronounced hyperattenuation of the vessel.

On the postinterventional VNC series, significantly higher attenuation was found in clotted than in perfused arteries, but not on the DEw series. In 9 of 10 cases $(90.0 \%)$, residual clots could be identified by the blinded rater due to pronounced hyperattenuation of the vessel. On the IM series, no significant difference was found between clotted and perfused arteries. Whereas between the DEw and VNC series, no significant difference in attenuation was found in the clotted arteries, significantly lower attenuation was found on the DEw series than on the VNC series in perfused arteries (Table 3 and Fig 2).

There was a weak, not statistically significant positive correlation between hematocrit values and perfused arteries on the VNC 
series (Spearman $\rho, 0.24 ; 95 \%$ confidence interval $[\mathrm{CI}], 0.31-$ 0.66; $P>.05)$, but not on the other series.

On the VNC series, residually clotted vessels were identified in 9 of 10 arteries (90\%) due to vascular hyperattenuation, with a false-negative evaluation in 1 case. In the 6 patients with no residual clot, the absence of a residual clot was correctly detected in 5 cases, with 1 false-positive evaluation. The sensitivity for the detection of residual clots was thus $90 \%$ (95\% CI, 54.1\%-99.5\%), specificity was $83.3 \%$ (95\% CI, 36.5\%-99.1\%), positive predictive value was $63.5 \%$ (95\% CI, 35.9\%-83.7\%), and the negative predictive value was $37.5 \%$ (95\% CI, 16.2\%-64.1\%). The accuracy was $87.5 \%$.

The Cohen $\kappa$ for the evaluation of the interrater agreement for the localization of initial clots was 0.762 (95\% CI, 0.324-1.0), and for the localization of residual clots, 0.733 (95\% CI, 38.9-1.0). The intraclass correlation coefficient regarding the measurements (Table 4) of the initial clots was $0.8196(P<.05)$, and for the measurements of residual clots in the VNC map, $0.9133(P<.05)$.

\section{DISCUSSION}

This study demonstrated that it is possible to detect intra-arterial clots shortly after the administration of intra-arterial contrast agent by DECT with quantitative measurements and subjective clot detections. Sensitivity and specificity were high, and the interrater agreement was good. On the DEw series, clotted and perfused vessels displayed a similar attenuation, but on the VNC series, clotted arteries were more attenuated than perfused arteries.

In various earlier studies, DECT in combination with the Brain Hemorrhage software has been described as a useful tool for differentiating intracranial hemorrhage and contrast staining after prior intravenous contrast application ${ }^{13,14,17}$ or neuroendovascular therapy. ${ }^{15,18,19}$

It has been shown that by using a combined evaluation of the VNC and IM series from DECT, sensitivity and specificity for the

\begin{tabular}{|c|c|c|}
\hline & \multicolumn{2}{|c|}{$P$ Values (Wilcoxon) } \\
\hline & Clots & Vessels \\
\hline DEw and VNC & .5703 & $.0020^{a}$ \\
\hline DEw and IM & $.0020^{a}$ & $.0020^{a}$ \\
\hline VNC and IM & $.0020^{a}$ & $.0020^{a}$ \\
\hline
\end{tabular}

a Significant difference.

Clots
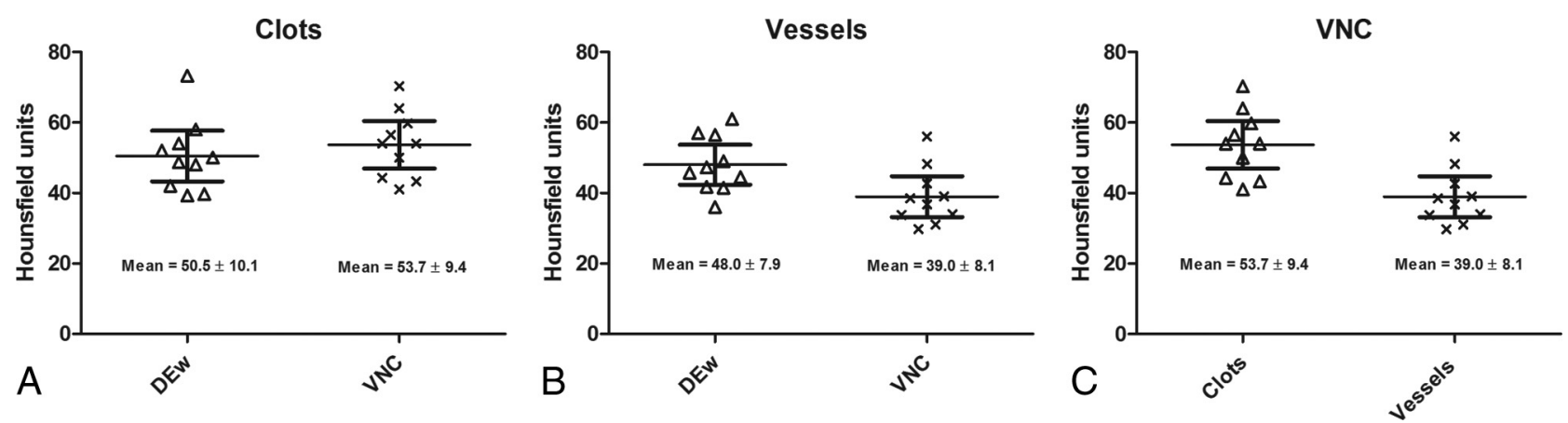

detection of an underlying contrast-enhancing mass within a cerebral hemorrhage was as high as $94.4 \%$ and $97.4 \%,{ }^{17}$ with positive or negative predictive values up to $95.7 \%$ and $94.1 \% .{ }^{30}$ With regard to the differentiation between hemorrhage and contrast staining in different intracranial compartments, another study even described a sensitivity of $100 \%$, specificity between $84.4 \%$ and $100 \%$, and accuracy between $87.2 \%$ and $100 \%$, with some limitations in calcified areas. ${ }^{14}$ For the differentiation between hemorrhage and contrast staining after endovascular stroke therapy, it has been suggested that sensitivity, specificity, and accuracy are $100 \%, 91 \%$, and $93 \%$, respectively, ${ }^{13}$ with positive and negative predictive values of up to $100 \%$ and $89 \% .{ }^{18,19}$ In the present study, a sensitivity of $90 \%$, specificity of $83 \%$, a positive predictive value of $63 \%$, and a negative predictive value of $38 \%$ were reached. The accuracy was $87.5 \%$. These values for sensitivity, specificity, and accuracy are of the same magnitude as those reported for the differentiation between intraparenchymal hematomas and intraparenchymal contrast staining ${ }^{13,18,19}$ or for the differentiation between these features at other locations. ${ }^{31}$ However, the positive and negative predictive values are clearly lower than those for the differentiation between intraparenchymal hematomas and intraparenchymal contrast staining, for example. The reason for this difference is most likely the small size of clots detected in this study. In contrast to intraparenchymal spaceoccupying hematomas, partial volume effects that lower the attenuation of the clots and make them more difficult to detect could play a role in the evaluation of residual intra-arterial clots. In this study, angiography as the criterion standard method was used for comparison, but in the other studies, only follow-up tests were available as the standard of reference. ${ }^{13,14,18,19}$

Table 4: Results from attenuation measurements of raters 1 and 2 and intraclass correlation on the different series

\begin{tabular}{cccc}
\hline & Rater 1 (Mean) & Rater 2 (Mean) & $\begin{array}{c}\text { Intraclass } \\
\text { Correlation }\end{array}$ \\
\hline Clot & & & \\
CT & $58.88 \pm 10.98$ & $56.57 \pm 9.49$ & 0.8244 \\
DEw & $50.50 \pm 10.08$ & $54.53 \pm 25.30$ & 0.7599 \\
VNC & $53.72 \pm 9.42$ & $54.43 \pm 8.33$ & 0.9133 \\
IM & $16.02 \pm 12.07$ & $19.60 \pm 36.08$ & 0.65 \\
Vessel & & & \\
CT & $43.35 \pm 5.54$ & $43.99 \pm 5.74$ & 0.8779 \\
DEw & $49.37 \pm 7.44$ & $50.68 \pm 10.05$ & 0.7687 \\
VNC & $41.64 \pm 7.87$ & $46.23 \pm 5.63$ & 0.6277 \\
IM & $11.22 \pm 6.90$ & $10.88 \pm 11.35$ & 0.6682 \\
\hline
\end{tabular}

Note:- CT indicates preinterventional CT.

FIG 2. Mean values with upper/lower $95 \%$ confidence intervals and SDs of Hounsfield attenuation measured in clotted $(A)$ and perfused $(B)$ vessels in the weighted dual-energy and virtual noncontrast series and as a comparison of clotted and perfused vessels in the VNC series (C). 
The $\kappa$ value of 0.733 for interrater agreement with respect to the detection of residual clots was of the same magnitude as the $\kappa$ value for the preinterventional detection of the initial clots $(\kappa=$ 0.762 ). The interrater agreement can thus be considered good. ${ }^{28}$ The intraclass correlation coefficient for the preinterventional and residual clot attenuation (Table 3) can be considered strong. ${ }^{29}$ The good detectability of residual clots may be due to the difference between the attenuation of the clots and the perfused vessels with only a small overlap (Fig 2). Both indicate that the method can be readily reproduced.

Only 1 existing study describes the application of DECT for the differentiation between intra-arterial contrast agent and intraarterial cerebral clots in 10 patients as a co-result. ${ }^{14}$ However, no information about the location of or the reason for the investigated clots was given in this study, and no ROI measurements were performed as in our study. Moreover, in the study mentioned, the lumens of branches involved were not visualized and the respective lesions were not associated with reported clinical symptoms, so the observation must be considered unverified or anecdotal.

Thus far, no studies are available that describe the use of DECT to detect residual peripheral intra-arterial clots after endovascular therapy, to our knowledge.

The described method of clot detection after intravascular contrast administration has various potential clinical applications. After endovascular stroke therapy with angiographically proved residual clots, DECT could be used to demonstrate delayed recanalization or early reclotting of initially recanalized arteries. Early postinterventional DECT could therefore lead to consequences for treatment, such as modification of medication or transfer to an intensive care unit.

In addition to this application, the described technique could be applied to detect early thromboembolic complications after other neurointerventional procedures with persistent contrast staining such as intracranial stent placement or coiling. In addition, it could be useful in cases with cerebral thromboembolic complications after interventional procedures of other regions such as the heart or the aortic arch. Another potential application could be in patients with suspected arterial or even venous intracerebral clots after intravenous contrast application for various reasons without the requirement of additional contrast agent application.

In this study, there was a low but not significant correlation between hematocrit values and perfused arteries on the VNC series. However, in general, the hematocrit levels seem to have only little impact on vascular attenuation before or after endovascular therapy, in contrast to the findings in other studies. ${ }^{26,27}$ One reason for this phenomenon might be that some of the hematocrit levels were determined before endovascular therapy, and others, immediately afterward. Therefore a dilution effect due to catheter flushing or intravenous fluid application might play a role. In addition, the relatively small population in this study might inadvertently mask the effect of hematocrit on arterial attenuation.

Several limitations of this study must be mentioned. Due to the occasionally small size of the peripheral, residually clotted, hyperattenuated arteries, it was not always easy to distinguish them from image noise. As discussed above, it has to be assumed that the size of a structure has a relevant impact on the applicability of the method to distinguish tissues, with better distinguishability between tissues in larger hemorrhages or clots, though no publication is available regarding this topic now. Complementary studies with larger patient populations must be conducted to elucidate not only the influencing factors vessel size, clot volume, and vessel direction on the sensitivity, specificity, and positive or negative predictive value of DECT but also the effect of technical factors such as mode of acquisition (axial or helical), signal-tonoise ratio, section thickness, or postprocessing of smaller FOVs and thinner sections. Furthermore, an estimation of the degree of a stenosis or the differentiation between stenosis and occlusion, such as might be possible with contrast-enhanced imaging, is not feasible with the described method. Due to the small number of patients, the CIs for sensitivity, specificity, positive predictive value, negative predictive value, and accuracy are relatively wide. They must be determined more precisely in further studies.

\section{CONCLUSIONS}

The described technique makes it possible to accelerate standardized stroke management of patients with previous contrast administration in whom intracranial clot detection with nonenhanced CT would be otherwise difficult. The application of DECT after contrast administration described here can make additional time-consuming imaging studies such as MR imaging or the application of additional contrast agent unnecessary.

Disclosures: Elke R. Gizewski-UNRELATED: Payment for Lectures (including service on Speakers Bureaus): Bracco Imaging, lecture for radiologists-in-training.

\section{REFERENCES}

1. Novakovic RL, Toth G, Narayanan S, et al. Retrievable stents, "stentrievers," for endovascular acute ischemic stroke therapy. Neurology 2012;79:S148-57

2. Przybylowski CJ, Ding D, Starke RM, et al. Evolution of endovascular mechanical thrombectomy for acute ischemic stroke. World J Clin Cases 2014;2:614-22

3. Chiro GD, Brooks RA, Kessler RM, et al. Tissue signatures with dualenergy computed tomography. Radiology 1979;131:521-23

4. Millner MR, McDavid WD, Waggener RG, et al. Extraction of information from CT scans at different energies. Med Phys 1979;6:70-71

5. Genant HK, Boyd D. Quantitative bone mineral analysis using dual energy computed tomography. Invest Radiol 1977;12:545-51

6. Johnson TR. Dual-energy CT: general principles. AJR Am J Roentgenol 2012;199:S3-8

7. Kamiya K, Kunimatsu A, Mori H, et al. Preliminary report on virtual monochromatic spectral imaging with fast $\mathrm{kVp}$ switching dual energy head CT: comparable image quality to that of $120-\mathrm{kVp} \mathrm{CT}$ without increasing the radiation dose. Jpn J Radiol 2013;31:293-98

8. Thomas C, Krauss B, Ketelsen D, et al. Differentiation of urinary calculi with dual energy CT: effect of spectral shaping by high energy tin filtration. Invest Radiol 2010;45:393-98

9. Zhang LJ, Peng J, Wu SY, et al. Dual source dual-energy computed tomography of acute myocardial infarction: correlation with histopathologic findings in a canine model. Invest Radiol 2010;45:290-97

10. Chae EJ, Seo JB, Lee J, et al. Xenon ventilation imaging using dualenergy computed tomography in asthmatics: initial experience. Invest Radiol 2010;45:354-61

11. Kalender WA. Computertomographie. Erlangen: Publicis; 2006

12. Simpkin DJ. Radiation interactions and internal dosimetry in nuclear medicine. Radiographics 1999;19:155-67; quiz 153-54

13. Gupta R, Phan CM, Leidecker C, et al. Evaluation of dual-energy CT 
for differentiating intracerebral hemorrhage from iodinated contrast material staining. Radiology 2010;257:205-11

14. Phan CM, Yoo AJ, Hirsch JA, et al. Differentiation of hemorrhage from iodinated contrast in different intracranial compartments using dual-energy head CT. AJNR Am J Neuroradiol 2012;33:1088-94

15. Brockmann C, Scharf J, Nolte IS, et al. Dual-energy CT after periinterventional subarachnoid haemorrhage: a feasibility study. Clin Neuroradiol 2010;20:231-35

16. Postma AA, Hofman PA, Stadler AA, et al. Dual-energy CT of the brain and intracranial vessels. AJR Am J Roentgenol 2012;199: S26-33

17. Kim SJ, Lim HK, Lee HY, et al. Dual-energy CT in the evaluation of intracerebral hemorrhage of unknown origin: differentiation between tumor bleeding and pure hemorrhage. AJNR Am J Neuroradiol 2012;33:865-72

18. Morhard D, Ertl L, Gerdsmeier-Petz W, et al. Dual-energy CT immediately after endovascular stroke intervention: prognostic implications. Cardiovasc Intervent Radiol 2014;37:1171-78

19. Tijssen MP, Hofman PA, Stadler AA, et al. The role of dual energy CT in differentiating between brain haemorrhage and contrast medium after mechanical revascularisation in acute ischaemic stroke. Eur Radiol 2014;24:834-40

20. Kraśnicki T, Podgórski P, Guziński M, et al. Novel clinical applications of dual energy computed tomography. Adv Clin Exp Med 2012;21:831-41

21. Karçaaltıncaba M, Aktas A. Dual-energy CT revisited with multidetector CT: review of principles and clinical applications. Diagn Interv Radiol 2011;17:181-94
22. De Cecco CN, Darnell A, Rengo M, et al. Dual-energy CT: oncologic applications. AJR Am J Roentgenol 2012;199:S98-105

23. Nicolaou S, Liang T, Murphy DT, et al. Dual-energy CT: a promising new technique for assessment of the musculoskeletal system. AJR Am J Roentgenol 2012;199:S78-86

24. Rutgers DR, van der Grond J, Jansen GH, et al. Radiologic-pathologic correlation of the hyperdense middle cerebral artery sign: a case report. Acta Radiol 2001;42:467-69

25. Leary MC, Kidwell CS, Villablanca JP, et al. Validation of computed tomographic middle cerebral artery "dot"sign: an angiographic correlation study. Stroke 2003;34:2636-40

26. Rauch RA, Bazan C, 3rd, Larsson EM, et al. Hyperdense middle cerebral arteries identified on CT as a false sign of vascular occlusion. AJNR Am J Neuroradiol 1993;14:669-73

27. Black DF, Rad AE, Gray LA, et al. Cerebral venous sinus density on noncontrast CT correlates with hematocrit. AJNR Am J Neuroradiol 2011;32:1354-57

28. Landis JR, Koch GG. The measurement of observer agreement for categorical data. Biometrics 1977;33:159-74

29. Shrout PE, Fleiss JL. Intraclass correlations: uses in assessing rater reliability. Psychol Bull 1979;86:420-28

30. Watanabe Y, Tsukabe A, Kunitomi Y, et al. Dual-energy CT for detection of contrast enhancement or leakage within high-density haematomas in patients with intracranial haemorrhage. Neuroradiology 2014;56:291-95

31. Flors L, Leiva-Salinas C, Norton PT, et al. Imaging follow-up of endovascular repair of type $B$ aortic dissection with dual-source, dualenergy CT and late delayed-phase scans. $J$ Vasc Interv Radiol 2014;25:435-42 